\title{
Funcionamiento familiar y depresión en adolescentes de la I.E. Zarumilla - Tumbes, 2013
}

\section{Family functioning and adolescent depression at Zarumilla School - Tumbes, 2013}

\author{
Marleny Torrel ${ }^{1}$, Mubbia Delgado ${ }^{2}$ \\ http://dx.doi.org/10.21503/CienciayDesarrollo.2014.v17i1.06
}

\section{RESUMEN}

La familia es la base fundamental de la sociedad en ella se dan los primeras conductas, el apego, amor y proporcionan un medio para la crianza de los hijos. Una familia disfuncional puede convertirse en una fuente de obstáculos en la adaptabilidad del adolescente, este grupo poblacional presenta una gran inestabilidad personal, emocional, por los cambios físicos, psicológicos y sociales que atraviesan, estos cambios puede llegar a originar problemas sociales que a menudo se observa en nuestra localidad. En la región Tumbes observamos que el 20,4\% presenta depresión, el 29\% de adolescentes ha pensado suicidarse en alguna ocasión, el 3,6\% lo ha intentado y el 29\% lo volvería hacer. Sabiendo que existe una posible asociación entre el funcionamiento familiar y la depresión y no existiendo estudios al respecto en nuestra región Tumbes, el propósito del presente estudio es determinar dicha asociación en el departamento. El estudio concluye en que la percepción de los niveles de funcionalidad familiar y su relación con la presencia o ausencia de depresión en los adolescentes presenta relación significativa en la muestra estudiada.

Palabras clave: Funcionalidad familiar, Depresión en adolescentes, Test FFSIL, Test de Zung.

\section{ABSTRACT}

The family is the fundamental basis of society, in it, the first behavior, attachment and love are given and provide a means for parenting. A dysfunctional family can become a source of obstacles in the adaptability of teenager, this population group has a great personal and emotional instability for physical, psychological and social changes which they feel; these changes may even cause social problems that often can be seen in our town. In the Tumbes region observed that $20.4 \%$ have depression, $29 \%$ of teenagers have thought suicide on occasion, $3.6 \%$ have tried it and $29 \%$ do it again. Knowing that there is a possible association between family functioning and depression and there are no studies in our Tumbes region, the purpose of this study was to determine the association in the department. The study concludes that the perception levels of family functioning and its relationship to the presence or absence of depression in teenagers have significant relationship in the studied sample.

Keywords: Family functioning, depression in teenagers, FFSIL test, Zung test.

\footnotetext{
1 Licenciada en Enfermería. Magister en Investigación y Pedagogía Universitaria. Directora de Escuela de Enfermería y Psicología UAP - Tumbes. E-mail: karytorrel@gmail.com

2 Bachiller en Enfermería UAP
} 


\section{INTRODUCCIÓN}

Un serio desafío que afronta el país es la formación de familias con carencia de alguna de las figuras progenitoras, ya que esta situación pone en riesgo la cohesión, cuidado, orientación y adaptación al medio de los hijos, sobre todo de los adolescentes que enfrentan diferentes cambios físicos, psicológicos y sociales.

En el Perú la población adolescente comprendida entre 10 a 19 años representa el 20\% del total de habitantes; es conocido que en esta etapa la persona atraviesa una serie de cambios biológicos, psicológicos y sociales, lo que predispone a cambios constantes de conducta, entre ellos: la agresividad, depresión, ansiedad y aislamiento, que puede llevarlos a un desequilibrio en la dinámica familiar. La falta de relación familiar trae consigo problemas en la adaptabilidad del adolescente en su entorno familiar, lo que no permite en él afrontar situaciones de depresión o estrés, orillándolo muchas veces a tomar decisiones equívocas. En la región Tumbes observamos que el 20,4\% presenta depresión, el 29\% de adolescentes ha pensado suicidarse en alguna ocasión, el 3,6\% lo ha intentado y el 29\% lo volvería hacer.

La depresión es un trastorno mental común que para el año 2020 va hacer uno de los principales problemas de salud pública. Esta depresión trae consigo múltiples problemas como: falta de ánimo, pérdida de interés, placer, sentimientos de culpa, baja autoestima, trastornos de sueño, falta de apetito, energía y de concentración, que pueden convertirse en crónicos conduciendo a un perjuicio sustancial si no se recurre a adecuadas terapias o manejo de estos cambios de conducta.

La adolescencia que se caracteriza por un continuo crecimiento, pues es la transición entre la infancia y edad escolar a la edad adulta; los cambios de cuerpo y mente provienen no solamente del individuo mismo, sino que se conjuga con su entorno, el cual es trascendental para que los grandes cambios psicológicos que se producen hagan que llegue a la edad adulta. Estos cambios son desarrollados y favorecidos gracias a la buena comunicación y cohesión familiar, la satisfacción de las necesidades afectivo-emocionales y materiales de sus miembros y estableciendo y manteniendo un equilibrio que sirva para enfrentar las tensiones que se producen en el curso del ciclo vital.

Una familia disfuncional puede convertirse en una fuente de obstáculos en la adaptabilidad del adolescente, este grupo poblacional presenta una gran inestabilidad personal, emocional, por los cambios físicos, psicológicos y sociales que atraviesa, estos cambios pueden llegar a originar problemas sociales que a menudo se observa en nuestra localidad, como la delincuencia, alcoholismo, drogadicción, pandillaje entre otros. Es necesario poner atención en los adolescentes y la familia en la cual se están desarrollando, para poder tener un análisis que permita formular nuevas estrategias y actividades dentro de los programas de atención integral del adolescente.

Sabiendo que existe una posible asociación entre el funcionamiento familiar y la depresión y no existiendo estudios al respecto en nuestra región Tumbes, el propósito del presente estudio es determinar dicha asociación, a fin de tener una base científica que nos permita más adelante implementar proyectos de intervención que aborden tal problemática.

\section{MATERIAL Y MÉTODOS}

Se utilizó el método hipotético - deductivo, el diseño del estudio fue observacional, transversal, correlacional. La muestra estuvo conformada por 88 adolescentes de la Institución Educativa Mixta Zarumilla con edades comprendidas entre los 15 y 18 años. Se aplicó el Test de Funcionamiento Familiar (FFSIL), que consiste en medir 
el grado de funcionalidad basado en 7 variables: cohesión, armonía, comunicación, afectividad, roles, adaptabilidad y permeabilidad, dicho test permitió identificar el factor relacionado causante del problema familiar. Así mismo se aplicó el Test de Zung, que es una escala de autoevaluación para la depresión, el cual nos permitió evaluar el nivel de depresión en los alumnos, a través de las características más comunes de la depresión, tales como: el efecto dominante, los equivalentes fisiológicos, otras perturbaciones y las actividades psicomotoras.

Para medir la confiabilidad de los instrumentos se aplicó el Alfa de Cronbach, toda vez que los instrumentos son de tipo escala Likert. El Alfa de Cronbach para el Test de Zung fue de $\alpha=0,8$ y para el Test FFSIL el valor fue de $\alpha=0,97$; dichos resultados nos confirman que ambos test presentan alta confiabilidad y sea cuales fueran las oportunidades en que se aplique, en las mismas condiciones, siempre obtendremos los mismos resultados.

\section{RESULTADOS}

Para el funcionamiento familiar, luego de aplicar el test FFSIL se observa que no se encontraron familias totalmente funcionales, el $26,1 \%$ de los estudiantes provenían de familias moderadamente funcionales, siendo de mayor porcentaje los alumnos provenientes de familias disfuncionales $60,2 \%$, finalmente un $13,6 \%$ de estudiantes pertenece a familias severamente disfuncionales (Tabla 1). Las diferencias estadísticas establecidas entre las proporciones son altamente significativas $(\mathrm{p}<0,05)$

Tabla 1: Funcionamiento familiar de los alumnos de la I.E.M. Zarumilla, 2013.

\begin{tabular}{lccc}
\multicolumn{1}{c}{ Funcionamiento familiar } & Frecuencia & Porcentaje & P - valor chi ${ }^{2}$ \\
Familia moderadamente funcional & 23 & 26,1 & \\
Familia disfuncional & 53 & 60,2 & \multirow{2}{*}{2,5 E-7 } \\
Familia severamente disfuncional & 12 & 13,6 & \\
Total & 88 & 100,0 & \\
\hline
\end{tabular}

Fuente: Test FFSIL aplicado a la muestra estudiada.

Respecto al Índice de depresión, luego de aplicar el Test de Zung se observan adolescentes normales en un $59,1 \%$ y adolescentes ligeramente y moderadamente deprimidos en el 29,5 y $11,4 \%$ respectivamente (Tabla 2). Las diferencias estadísticas establecidas entre las proporciones son altamente significativas $(\mathrm{p}<0,05)$

Tabla 2: Depresión en los alumnos de la I.E.M. Zarumilla, 2013.

\begin{tabular}{lccc}
\multicolumn{1}{c}{ Depresión } & Frecuencia & Porcentaje & P - valor chi ${ }^{2}$ \\
Normal & 49 & 55,7 & \\
Ligeramente deprimido & 29 & 33,0 & \multirow{2}{*}{$2,34 \mathrm{E}-6$} \\
Moderadamente deprimido & 10 & 11,4 & \\
Total & 88 & 100,0 & \\
\hline
\end{tabular}

Fuente: Test de Zung aplicado a la muestra estudiada. 
Se consideró también establecer los niveles depresión son diferentes en hombres que en de depresión de acuerdo al género de los mujeres $(\mathrm{p}<0,05)$, siendo el género femenino participantes obteniendo que los niveles de mucho más propenso a esta patología. Tabla 3.

Tabla 3: Depresión por género en los alumnos de la I.E.M. Zarumilla, 2013.

\begin{tabular}{|c|c|c|c|c|c|c|}
\hline & & Nivel d & depresión en la & adolescentes & & p - valor \\
\hline & & Normal & $\begin{array}{l}\text { Ligeramente } \\
\text { deprimido }\end{array}$ & $\begin{array}{c}\text { Moderadamente } \\
\text { deprimido }\end{array}$ & Total & $\begin{array}{c}\text { Mann } \\
\text { Whitney }\end{array}$ \\
\hline & & 13 & 24 & 6 & 43 & \\
\hline & & $26,53 \%$ & $82,76 \%$ & $60,00 \%$ & $48,86 \%$ & \\
\hline Sexo & Masculino & 36 & 5 & 4 & 45 & \\
\hline & Masculino & $73,47 \%$ & $17,24 \%$ & $40,00 \%$ & $51,14 \%$ & $2,54 \mathrm{E}-5$ \\
\hline & & 49 & 29 & 10 & 88 & \\
\hline Total & & $100,00 \%$ & $100,00 \%$ & $100,00 \%$ & $100,00 \%$ & \\
\hline
\end{tabular}

Fuente: Test de Zung aplicado a la muestra estudiada.

Finalmente se estableció la asociación entre funcionamiento familiar y nivel de depresión en los adolescentes evaluados, observando de acuerdo a la Tabla 4 que existe asociación significativa $(\mathrm{p}<0,05)$ entre las variables estudiadas. Gran porcentaje de los alumnos pertenecientes a familias disfuncionales y severamente disfuncionales acusan depresión ligera y/o moderada.

Tabla 4: Asociación entre Funcionamiento familiar y nivel de depresión en los alumnos de la I.E.M. Zarumilla, 2013.

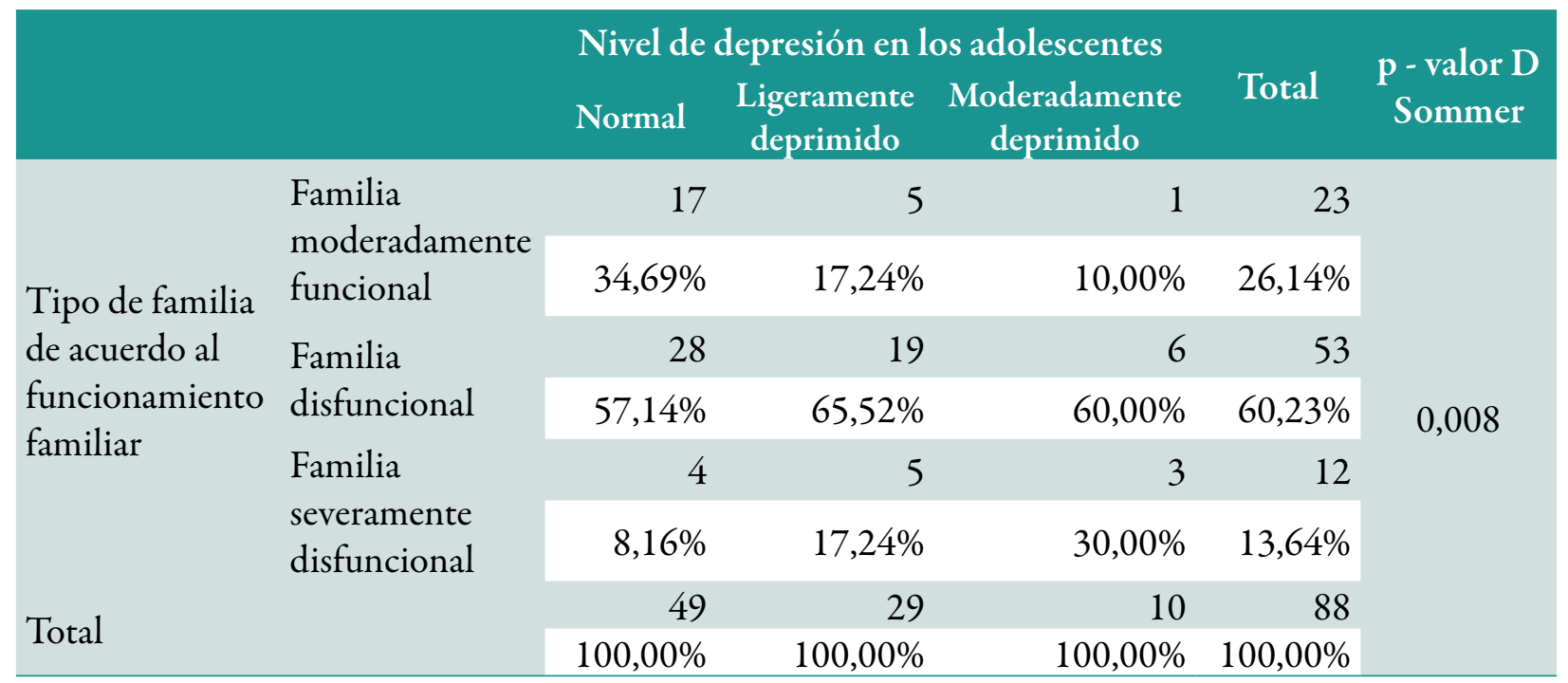

Fuente: Test FFSIL y Test de Zung aplicado a la muestra estudiada. 


\section{DISCUSIÓN}

$\mathrm{Al}$ investigar sobre el funcionamiento familiar y la depresión en los adolescentes y los problemas que pueden atravesar a nivel personal o social, es importante tomar en cuenta la influencia de la familia ya que la funcionalidad familiar en esta etapa no solo depende de cómo reaccionan los hijos sino también de la forma en cómo los padres o apoderados del menor lo percibén, comprenden y asumen esta etapa de la vida. En esta investigación el resultado indica que hay relación significativa entre la presencia o ausencia de depresión y la percepción de funcionalidad familiar en los adolescentes estudiados.

En nuestro estudio encontramos una alta prevalencia de familias disfuncionales, este resultado difiere de lo encontrado por Mendoza (2006) en una población de estudiantes mexicanos, en dicho estudio las proporciones encontradas fueron de $52 \%$, 39\% y $9 \%$ para familias moderadamente funcionales, moderadamente disfuncionales y severamente disfuncionales, lo que contrasta con el 26,1\%; 60,2\% y 13,6\% encontrado en nuestro estudio, al realizar el estadístico chi cuadrado podemos observar que las diferencias de las proporciones entre ambos estudios son altamente significativas $(\mathrm{p}<0,05)$. Cabe resaltar que a diferencia del estudio de Mendoza el estudio que presentamos fue realizado en una institución educativa de estrato bajo, que aunque no es una variable estudiada, podría influir en los resultados, ya que la falta de recursos económicos podría desencadenar el abandono familiar y el inicio en hábitos no saludables por parte de los cónyuges responsables del hogar.

Los grados de depresión en los estudiantes también difieren de una población mexicana de la misma edad Zapata (2007), mientras que para este autor los grados de depresión son de 66,9\%; $27,2 \%$ y 5,9 para adolescentes normales, ligeramente deprimidos y moderadamente deprimi- dos, los porcentajes en nuestro estudio fueron de $55,7 \%, 33 \%$ y $11,4 \%$. Las diferencias entre proporciones fueron ligeramente significativas $(\mathrm{p}<$ $0,05)$.

$\mathrm{Al}$ establecer diferencias por género respecto a los niveles de depresión se observa que la mayoría de adolescentes son mujeres y de ellas el $82,8 \%$ tienen indicador de depresión ligera, porcentaje que supera a los varones con depresión, cuyo porcentaje es de 17,2\%; asimismo, la depresión moderada en mujeres es del $60 \%$, valor que supera al $40 \%$ de los hombres. Estos datos son consecuentes con diversas investigaciones realizadas (Callabed y cols 1997, Parra 2007, Álvarez y cols 2009) donde se observa que las mujeres tiene mayor tendencia a la depresión que los varones, por los indicadores evidentes de tristeza, llanto fácil, desánimo y todo signo emocional, en cambio en los varones el aspecto emocional está más controlado o menos evidente, esta información nos permite abordar a los pacientes adolescentes conociendo sus indicadores cognitivos y el encubrimiento de sus sentimientos.

Existen discrepancias en cuanto a la asociación o no de las variables de estudio; tanto la investigación de Zapata y Cols. (2007) como el estudio de Cogollo (2009) indican que no se encontró relación significativa en las variables; no obstante, en las investigaciones dadas por Álvarez y cols. (2009) y Leyva y cols (2007), se encuentran cierta asociación entre ellas. Asimismo, en investigaciones que tratan el tema de depresión en adolescentes suelen informar que uno de los factores a tomar en cuenta es la funcionalidad familiar, por otro lado cuando se estudia la disfuncionalidad familiar en adolescentes también se tiende a mencionar conductas asociadas con rasgos depresivos en ellos sin llegar a definir una asociación directa sino resaltando que ambas variables siguen siendo de interés para el contexto en que el adolescente se desarrolla. En nuestro estudio claramente encontramos una asociación 
entre funcionalidad familiar y niveles de depresión en los estudiantes evaluados; coincidimos con Álvarez, Ramírez y Cols (2009) quienes indican que existe una relación inversamente proporcional entre los síntomas depresivos y los conflictos familiares, lo que sugiere que, a mayor índice de depresión existe menos cohesión familiar, demostrando que la carencia de apoyo social en el ambiente familiar del adolescente afecta en su proceso psicosocial.

Finalmente es importante aclarar que este estudio tomó en cuenta la depresión definida por el Test de Zung donde los indicadores se basan en factores psicológicos que ponen en evidencia conductas relacionadas al estado depresivo, no obstante existen casos en los que los adolescentes manifiestan conductas que reflejan agresividad, fracaso académico, consumo de drogas, etc. que puedan encubrir un episodio o trastorno depresivo; estos casos no formaron parte del estudio pero de acuerdo a los resultados puede tomarse en cuenta para futuras investigaciones.

\section{CONCLUSIONES}

La percepción de los niveles de funcionalidad familiar y su relación con la presencia o ausencia de depresión en los adolescentes presenta relación significativa en la muestra estudiada.

El funcionamiento familiar de las familias de las que provienen los adolescentes de la I. E. Zarumilla fue de $60,2 \%$ familias disfuncionales; $26,1 \%$ familias moderadamente funcional y $13,6 \%$ familias severamente disfuncional. El nivel de depresión presentado por los adolescentes de la I. E. Zarumilla fue de $55,7 \%$ normales, $33 \%$ depresión ligera y el $11,4 \%$ de depresión moderada. Las mujeres presentaron índices mayores de depresión con respecto a los hombres, mientras que la depresión ligera en mujeres fue del $82,8 \%$ y depresión moderada 60\%; en los hombres la depresión ligera fue del 17,2\% y depresión mo- derada $40 \%$, dichas diferencias fueron estadísticamente significativas.

\section{RECOMENDACIONES}

Para el estudio se evaluó la depresión en función al Test de Zung que permite identificar los indicadores de depresión; no obstante es importante tener en cuenta que el diagnóstico de depresión propiamente dicho siempre ha sido difícil de catalogar en los adolescentes por los diversos cambios emocionales que experimentan, por lo que se recomienda investigar a profundidad el comportamiento de los adolescentes y su relación con el entorno, para conocer si la depresión es una causal de su conducta.

Los resultados de este estudio son determinantes y si bien indican que hay relación entre las variables es importante profundizar en el porqué de dicho resultado. Continuar investigando cerca de los problemas que surgen en la adolescencia es vital para observar cómo se comporta la familia en estas situaciones. La meta es conocer más sobre la adolescencia y sus problemáticas para proponer planes de intervención de índole sistémico (a nivel personal, familiar y social) basado en evaluación, intervención psicoeducativa e intervención psicoterapéutica familiar.

\section{REFERENCIAS BIBLIOGRÁFICAS}

1. Alberto Iram Villa-Manzano. (2009). Magnitud de la disfunción familiar y depresión como factores de riesgo para intento de suicidio. Revista Médica del Instituto Mexicano de Seguro Social, 643-646.

2. Alicia, P. L. (2002). Estudio de la estructura semiológica con una metodología tetradimensional y de las situaciones familiares y laborales crónicas en la depresión de la mujer. . Madrid: Informe de Tesis Doctoral. Universidad Complutense de Madrid.

3. Catalina Gonzáles Forteza, L. R. (2003). Correlatos psicosociales de depresión, ideación 
e intento suicida en adolescentes mexicanos. Psicothema, 524-532.

4. Javier Zapata-Gallardo, M. F.-G. (2007). Depresión asociada a la disfunción familiar en la adolescencia. Artemisa, 295-301.

5. Jesús, C. M. (2003). Comunicación familiar y Bienestar Psicosocial en Adolescentes. Actas del VIII Congreso Nacional de Psicología Social (págs. 23 - 27). Valencia: Universidad de Valencia.

6. L dos Santos Palazzo, J. U.-F. (2001). Depresión en la adolescencia en centros de atención primaria: importancia de un problema oculto en salud colectiva. Atención primaria, 15-21.
7. Mendoza S, S. H. (2006). Análisis de la Dinámica Familiar y Funcionalidad GFamiliar en Atención Primaria. Archivos en Medicina Familiar, 27-32.

8. Mónica Álvarez Zuñiga, B. R. (2009). La relación entre depresión y conflictos familiares en adolescentes. International Journal of Psychology anf Psychological Therapy, 205-216.

9. Rafael Leya-Jiménez, A. M. (2007). Depresión en adlescentes y funcionamiento familiar. Revista Médica del Instituto Mexicano del seguro Social, 225-232. 